\title{
Local equation of state and velocity distributions of a driven granular gas
}

\author{
Olaf Herbst, Peter Müller, Matthias Otto, and Annette Zippelius \\ Institut für Theoretische Physik, Georg-August-Universität, D-37077 Göttingen, Germany
}

(Dated: April 9, 2004)

\begin{abstract}
We present event-driven simulations of a granular gas of inelastic hard disks with incomplete normal restitution in two dimensions between vibrating walls (without gravity). We measure hydrodynamic quantities such as the stress tensor, density and temperature profiles, as well as velocity distributions. Relating the local pressure to the local temperature and local density, we construct a local constitutive equation. For strong inelasticities the local constitutive relation depends on global system parameters, like the volume fraction and the aspect ratio. For moderate inelasticities the constitutive relation is approximately independent of the system parameters and can hence be regarded as a local equation of state, even though the system is highly inhomogeneous with heterogeneous temperature and density profiles arising as a consequence of the energy injection. Concerning the local velocity distributions we find that they do not scale with the square root of the local granular temperature. Moreover the high-velocity tails are different for the distribution of the $x$ - and the $y$-component of the velocity, and even depend on the position in the sample, the global volume fraction, and the coefficient of restitution.
\end{abstract}

PACS numbers: 45.70.-n, 51.30.+i, 51.10.+y

\section{INTRODUCTION}

The physics of vibro-fluidized granular materials is far from being fully understood. In particular, the applicability of hydrodynamics [1, 2, 3, 4, 5] is still an object of debate [6, 7, 8]. Despite the similarities to the hydrodynamics of elastic hardsphere systems, concerning e.g. the appearance of instabilities $[9,10,11,12]$, a main difference to ordinary fluids is the fact that continuous energy injection is vital to maintain a stationary state. Otherwise a gas of inelastic spheres would collapse, even in the absence of gravity. The non-trivial nature of this stationary state has been elucidated in experiments of vibrating grains. Among the most striking features found are non-Gaussian velocity distributions [13, 14, 15, 16] and cluster formation [17, 18, 19]. Furthermore, several attempts have been made to extract either an equation of state or more generally a scaling relation for the thermodynamic variables [20, 21].

The specification of the driving mechanism is a crucial ingredient for any model describing vibrated granular fluids. A rather simple approach, though less appealing from an experimental point of view, utilizes stochastic bulk heating by uncorrelated random forces, which act on every particle at every instant of time [22, 23, 24, 25]. In [26] random restitution coefficients were considered with a probability distribution allowing for values both smaller and greater than one. However, this yields non-universal properties depending on the specific form of this distribution. The multiplicative bulk driving defined in terms of stochastic collision rules in [27] is similar in spirit. As compared to bulk driving, a driving mechanism which acts only at a boundary of the system is much closer to experiments. In [2] the energy influx at the boundary has been modeled by a heuristically motivated ansatz for the heat current, while [28, 29] assume heating at the boundary through thermal walls. In the present paper the driving mechanism consists in incrementing a particle's velocity by a constant amount after every collision with a wall. This way of driving was used before in e.g. [30, 31], and with slight modifications in [32, 33, 34].
One theoretical approach to study granular systems at fluid densities rests on kinetic theory, see e.g. [5, 35, 36, 37] and references therein. Particular applications to driven granular gases can be found in [23, 26, 27, 28, 38]. Much of this work has been based on the Boltzmann equation, modified for inelastic collisions. One method to solve this nonlinear equation is based on the local equilibrium distribution [39], which is only known for the elastic case. For systems with strongly inelastic collisions, the stationary state is unknown so that a systematic discussion of transport properties within kinetic theory is severely hampered.

Hydrodynamic studies [2, 11, 12, 30] have been motivated partly by the search for an understanding of temperature and density profiles [2], but also by experiments on hydrodynamic-like instabilities [9]. In the hydrodynamic approach the question arises, how to relate the pressure to the density and temperature. Several equations of state for the global quantities have been proposed, either interpolating between the high and low density limit [2] or invoking the Boltzmann equation [31] for inelastically colliding particles. It is not clear, whether an equation of state also holds for the local hydrodynamic fields in a strongly driven, non-equilibrium system, where the fields are strongly inhomogeneous.

Most simulations of vibrated granular gases have been based either on event-driven molecular dynamics [31, 40, 41] or the direct-simulation Monte Carlo method [28, 30, 42, 43]. If the system is driven through the boundaries, inhomogeneous density and temperature profiles are measured. For low densities the computed temperature profiles agree well with hydrodynamic theory [2, 30], whereas for moderate or high densities the profiles are not well understood with the exception of almost elastically colliding particles. The full stress tensor, including potential contributions, has only been computed for freely cooling systems [44]. In simulations of driven granular gases [28] the collisional part of the stress tensor has not been measured, and in [31] the stress tensor is not measured directly but instead computed from a local equation of state.

Inspired by experiment [16], a lot of emphasis has been put 
on the tails of the velocity distribution functions, which were found to be overpopulated as compared to a Maxwellian. In fact, all intermediate types of decay between a Gaussian and an exponential were observed [31, 42, 45, 46]. In addition mixtures [31, 47, 48] and rough spheres [49] have been investigated as well as hydrodynamic instabilities such as convection [50, 51] and pattern formation [52].

In this paper, we present results from event-driven simulations of inelastic spheres in two dimensions confined between two vibrating walls without gravity. Our focus is on the stationary state, which is reached when dissipation by particle collision equals energy injection due to the vibrating walls. In the stationary state, density and temperature profiles are shown to be strongly inhomogeneous due to the driving walls - even in a range of parameters, where clustering is only a minor effect. This has led us to

(a) derive a constitutive equation by relating the measured hydrodynamic fields, granular temperature $T(x)$, volume fraction $\phi(x)$ and pressure $p(x)$, at each point $x$ and

(b) check whether this constitutive equation is universal or depends on the global system parameters of the model, like the aspect ratio of the cell, the overall volume fraction or the coefficient of restitution of the disks. For moderately inelastic systems ( $\alpha=0.9)$ the constitutive equation is (almost) independent of the remaining global system parameters so that the constitutive equation can be interpreted as a local equation of state for the a driven granular gas in the stationary state, even though the latter is highly inhomogeneous with heterogeneous temperature and density profiles. In contrast, for strongly inelastic systems $(\alpha=0.5)$ the constitutive equation depends significantly on the global system parameters so that the concept of a local equation of state cannot be sustained in this case.

We furthermore discuss the one-particle distribution function in the stationary state and show that

(c) the local distribution $f_{x}\left(x, v_{x}\right)$ of $v_{x}$, the velocity in the direction of driving, is not a function of the rescaled variable $v_{x} / \sqrt{T_{x}(x)}$ alone. Similarly, curves of $f_{y}\left(x, v_{y}\right)$ cannot be mapped onto a master curve for different $x$, when plotted against $v_{y} / \sqrt{T_{y}(x)}$. Here $T_{i}(x)$ denotes the local granular temperature associated with the translational motion in the $i$ direction. We find deviations from scaling at small and large arguments.

(d) The local velocity distributions $f_{x}$ and $f_{y}$ have highvelocity tails whose decay ranges from stretched exponential to almost Gaussian. The particular type of decay depends on the position in the sample, the overall particle density and the coefficient of restitution. Furthermore, the decay of $f_{x}$ for large velocities is generally different from that of $f_{y}$.

Taken together, one has to acknowledge that the stationary state of a driven granular gas is by no means universal. It shows peculiar features that depend on the precise values of the system parameters in contrast to the corresponding elastic system.

The outline of the paper is as follows. In Sec. II we introduce the model, specify the driving and define the observables. We briefly discuss balance of energy input through the walls and energy dissipation in binary collisions in Sec.

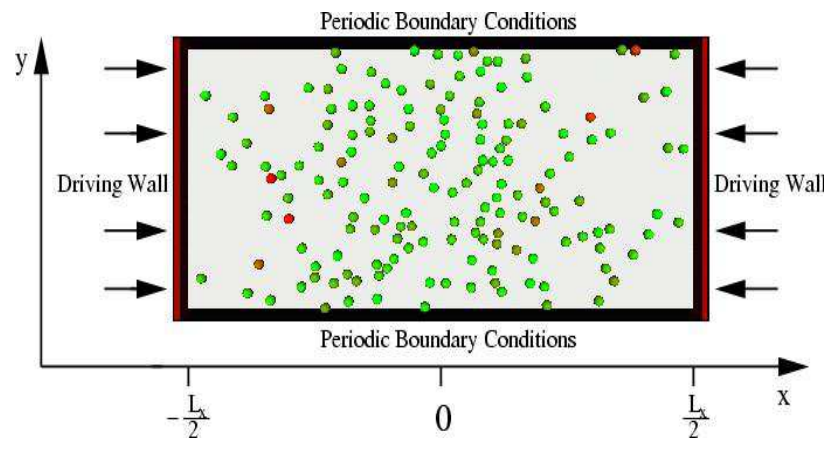

FIG. 1: Model of $\mathrm{N}$ disks, driven in the $x$-direction with periodic boundary conditions in the $y$-direction.

III Subsequently we present data for the profiles of the density, the temperature and the components of the stress tensor (Sec.IV). In Sec. $\nabla$ we relate the local density, pressure and temperature to derive "experimentally" an equation of state and check for its universality. Finally, in Sec. VI we discuss velocity distribution functions and their scaling behavior and present conclusions in Sec. VII

\section{MODEL AND OBSERVABLES}

We investigate a driven granular gas in 2 dimensions consisting of $N$ identical inelastic smooth hard disks of diameter $a$ and mass $m$ which are confined to a rectangular box with edges of length $L_{x}$ and $L_{y}$. The gas is driven through the walls perpendicular to the $x$-direction, which vibrate in an idealized saw-tooth manner (see below), while periodic boundary conditions are imposed in the $y$-direction. Fig. 1 shows a typical snapshot. The gas evolves in time through ballistic centerof-mass motion, binary inelastic collisions and particle-wall collisions.

\section{A. Binary collisions}

The inelastic nature of inter-particle collisions is the most important characteristic of granular media. As is often done we assume that it can be taken into account by a constant coefficient of normal restitution and briefly recall the collision rules for binary collisions, see e.g. [53, 54].

The unit-vector from the center of sphere two to the center of sphere one is denoted by $\hat{\boldsymbol{n}}:=\left(\boldsymbol{r}_{1}-\boldsymbol{r}_{2}\right) /\left|\boldsymbol{r}_{1}-\boldsymbol{r}_{2}\right|$ where $\boldsymbol{r}_{i}$ is the position vector of the center of mass of particle $i$. The center-of-mass velocities before a collision are denoted by $\boldsymbol{v}_{1}$ and $\boldsymbol{v}_{2}$, and the relative velocity by $\boldsymbol{v}_{12}=\boldsymbol{v}_{1}-\boldsymbol{v}_{2}$. Postcollisional quantities are primed. The relative velocity after a collision is assumed to obey

$$
\hat{\boldsymbol{n}} \cdot \boldsymbol{v}_{12}^{\prime}=-\alpha \hat{\boldsymbol{n}} \cdot \boldsymbol{v}_{12}
$$

where $\alpha \in[0,1]$ is the (constant) coefficient of normal restitution. The value $\alpha=1$ describes elastic collisions with energy 
conservation, while for $\alpha<1$ energy will not be conserved but decreased in each collision.

The constitutive equation (1) plus momentum conservation determines the post-collisional velocities for the disks

$$
\boldsymbol{v}_{1}^{\prime}=\boldsymbol{v}_{1}+\Delta \boldsymbol{v}_{\mathrm{pp}}, \quad \boldsymbol{v}_{2}^{\prime}=\boldsymbol{v}_{2}-\Delta \boldsymbol{v}_{\mathrm{pp}}
$$

where

$$
\Delta \boldsymbol{v}_{\mathrm{pp}}=-\frac{(1+\alpha)}{2}\left(\hat{\boldsymbol{n}} \cdot \boldsymbol{v}_{12}\right) \hat{\boldsymbol{n}}
$$

The model could easily be extended to rough spheres by also specifying the disks' moment of inertia as well as a coefficient of tangential restitution $\beta_{0}$ and Coulomb friction $\mu$ [55, 56], but here we restrict ourselves to smooth spheres so that rotational motion is decoupled from translational motion.

\section{B. Driving}

When a particle collides with a driving wall, energy is injected into the system. This can be modeled in different ways, for example by drawing a new velocity from a Maxwellian distribution of a given wall temperature [2] or by assuming that the wall has a coefficient of normal restitution that is greater than one. Both of these mechanisms have no close experimental equivalents, though. A more realistic model is to assume a vibrating wall moving either in a symmetric (e.g. sinusoidal) or in an asymmetric (e.g. saw-tooth) way. In addition, this can be combined with a normal (and also tangential) coefficient of restitution [33, 41, 57]. In this article we refrain from the latter and restrict ourselves to saw-tooth driving of the walls in the limit of vanishing amplitudes $A$ and diverging frequency $\nu$ such that $A \nu=: v_{\text {drive }} / 2$ is a constant. This ensures that the driving walls are always located at the same positions and leads to the following simple expression for a particle's change of velocity due to a collision with the left/right wall

$$
\boldsymbol{v}^{\prime}=\boldsymbol{v}+\Delta \boldsymbol{v}_{\mathrm{pw}} \quad \text { where } \quad \Delta \boldsymbol{v}_{\mathrm{pw}}=\left(-2 v_{x} \pm v_{\text {drive }}\right) \boldsymbol{e}_{x}
$$

and $\boldsymbol{e}_{x}$ stands for the unit vector in $x$-direction.

\section{Observables from simulations}

Following e.g. [58, 59] we performed event-driven simulations in two dimensions with periodic boundary conditions in the $y$-direction and two identical idealized vibrating elastic walls in the $x$-direction. We initialize the system by placing the disks on a triangular lattice with a Gaussian velocity distribution. To let the correlations of the initial state relax, the system is evolved elastically with periodic boundary conditions in the $y$-direction and elastic non-vibrating walls in the $x$-direction for an average of 100 collisions per particle. Then we switch on the driving of the left and right wall and the dissipation for particle-particle-collisions. Before we start measuring the observables we let the system relax further until, at time $t_{0}$, it has reached a stationary state, as indicated by the total kinetic energy, which fluctuates around a time-independent mean value.

To measure hydrodynamic fields in the stationary state we subdivide our box into cells $\mathcal{V}_{\boldsymbol{r}}$ of area $\left|\mathcal{V}_{\boldsymbol{r}}\right|$, centered at position $\boldsymbol{r}=(x, y)$. We then count the number of particles $f\left(\boldsymbol{r}, v_{x}, v_{y}, t\right)\left|\mathcal{V}_{\boldsymbol{r}}\right| d v_{x} d v_{y}$ at time $t$ in cell $\mathcal{V}_{\boldsymbol{r}}$ with an $x$-component of the velocity between $v_{x}$ and $v_{x}+d v_{x}$ and $y$-component of the velocity between $v_{y}$ and $v_{y}+d v_{y}$. Such a local observable fluctuates as a function of time. To eliminate these fluctuations we average over a (long) time interval of length $\tau$ and compute

$$
f_{\text {stat }}\left(\boldsymbol{r}, v_{x}, v_{y}\right):=\frac{1}{\tau} \int_{t_{0}}^{t_{0}+\tau} d t f\left(\boldsymbol{r}, v_{x}, v_{y}, t\right) .
$$

Of particular interest is the particle density

$$
\rho(\boldsymbol{r}):=\int_{\mathbb{R}} d v_{x} \int_{\mathbb{R}} d v_{y} f_{\text {stat }}\left(\boldsymbol{r}, v_{x}, v_{y}\right)
$$

or the area fraction $\phi(\boldsymbol{r}):=\rho(\boldsymbol{r}) \pi a^{2} / 4$ and the two components $i=x, y$ of the granular temperature

$$
T_{i}(\boldsymbol{r}):=\frac{m}{\rho(\boldsymbol{r})} \int_{\mathbb{R}} d v_{x} \int_{\mathbb{R}} d v_{y} f_{\text {stat }}\left(\boldsymbol{r}, v_{x}, v_{y}\right)\left(v_{i}-V_{i}(\boldsymbol{r})\right)^{2}
$$

where

$$
\boldsymbol{V}(\boldsymbol{r}):=\frac{1}{\rho(\boldsymbol{r})} \int_{\mathbb{R}} d v_{x} \int_{\mathbb{R}} d v_{y} f_{\text {stat }}\left(\boldsymbol{r}, v_{x}, v_{y}\right) \boldsymbol{v}
$$

is the velocity field. The total granular temperature in 2 dimensions is defined as $T(\boldsymbol{r}):=\left(T_{x}(\boldsymbol{r})+T_{y}(\boldsymbol{r})\right) / 2$.

The stress tensor at position $\boldsymbol{r}$ and time $t$ has a kinetic contribution and one that is due to the interactions between the particles $\boldsymbol{\sigma}(\boldsymbol{r}, t):=\boldsymbol{\sigma}^{\mathrm{kin}}(\boldsymbol{r}, t)+\boldsymbol{\sigma}^{\mathrm{int}}(\boldsymbol{r}, t)$. The kinetic part is given by

$$
\begin{aligned}
\sigma_{i j}^{\mathrm{kin}}(\boldsymbol{r}):=-m \int_{\mathbb{R}} d v_{x} & \int_{\mathbb{R}} d v_{y} f_{\text {stat }}\left(\boldsymbol{r}, v_{x}, v_{y}\right) \\
& \times\left[v_{i}-V_{i}(\boldsymbol{r})\right]\left[v_{j}-V_{j}(\boldsymbol{r})\right] .
\end{aligned}
$$

If the particles interact through (finite) forces the contribution due to interactions is given by the correlation of the particles' relative positions and the forces between them. For hard-core interactions there are no forces, and one has to consider the momentum transfer in a small time interval instead [59, 60]: Suppose there is a collision at time $t$ of particle $k$ with another particle, then the momentum change of particle $k$ will contribute to the stress tensor an amount proportional to $l_{i}^{k}(t) \Delta p_{j}^{k}(t)$. Here $l_{i}^{k}(t)$ is the $i$-th component of the vector of length $a / 2$ pointing from the center of disk $k$ to its collision contact point, and $\Delta p_{j}^{k}(t)$ is the $j$-th component of the momentum change of particle $k$ during this collision. To compute the collisional part of the stress tensor we need the total change of momentum in the time interval $[t-\Delta t, t]$ in the cell $\mathcal{V}_{\boldsymbol{r}}$ so that we have to keep track of all collisions $n$ occurring at times $t_{n} \in[t-\Delta t, t]$, for which at least one collision partner $k_{n}$ (i.e. its center of mass) is located in cell $\mathcal{V}_{\boldsymbol{r}}$ at time $t_{n}$ 
[59]

$$
\sigma_{i j}^{\mathrm{int}}(\boldsymbol{r}, t)=\frac{1}{\Delta t} \frac{1}{\left|\mathcal{V}_{\boldsymbol{r}}\right|} \sum_{t_{n}} \sum_{k_{n}} l_{i}^{k_{n}}\left(t_{n}\right) \Delta p_{j}^{k_{n}}\left(t_{n}\right) .
$$

The particle number $k_{n}$ of each such collision can take on one or two values, depending on whether one or both collision partners are located in cell $\mathcal{V}_{\boldsymbol{r}}$. Similar to the other hydrodynamic fields, in a second step $\boldsymbol{\sigma}^{\text {int }}(\boldsymbol{r}, t)$ is averaged over time in order to get the collisional part of the stress tensor in the stationary state

$$
\sigma_{i j}^{\mathrm{int}}(\boldsymbol{r})=\frac{1}{\tau} \int_{t_{0}}^{t_{0}+\tau} d t \sigma_{i j}^{\mathrm{int}}(\boldsymbol{r}, t) .
$$

The corresponding local pressure $p(\boldsymbol{r}):=-\operatorname{tr}(\boldsymbol{\sigma}(\boldsymbol{r})) / 2$ is defined as usual as the negative trace of the stress tensor divided by the space dimension.

Besides hydrodynamic fields we have also measured velocity distributions in Sec. VI] They are readily obtained by integrating out the relevant variables in the stationary-state distribution function $f_{\text {stat }}$. A different method, which is better suited to determine high-velocity tails, for example, will be presented in Sec. [VI below.

Coarse-grained measurements in space and time of certain observables may depend on the coarse-graining resolution. For example, this was demonstrated for the stress tensor in shear-flow driven granular systems in [60, 61]. Our measurements of observables in the stationary state, however, should not suffer from such effects for two reasons: First, we could not detect any significant non-zero local velocity field in the simulated systems and, second, because of the long-time average needed to obtain stationary-state quantities.

Moreover, in the simulations we have never found any significant dependence of the (long-time-averaged) hydrodynamic fields on $y$, the coordinate parallel to the driving walls. Yet, stripe states, which are homogeneous in $y$, but have an enhanced density in the middle of the sample, are known [10, 11] to exhibit instabilities with respect to density fluctuations in $y$. A marginal stability analysis [11] of granular hydrodynamics (for $\alpha$ close to one) determines the conditions under which such phenomena occur. As far as a comparison can be made, our systems fall into the stable region. Hence, we choose the cells $\mathcal{V}_{\boldsymbol{r}}$ as stripes along the $y$-direction, for which we write $\mathcal{V}_{x}$, and compute the hydrodynamic fields with spatial resolution in $x$-direction only. Thus, we also write $\rho(x)$ instead of $\rho(\boldsymbol{r})$ and change the notation accordingly for all other quantities. In the simulations the stripes $\mathcal{V}_{x}$ were all chosen to have equal width $L_{x} / 201$, the temporal resolution of our measurements was set to $\Delta t=1$, and the long-time average involves typically $10^{7}-10^{9}$ collision events.

Another instability of stripe states which is related to oscillations of the central dense cluster in $x$-direction [62] will be shortly addressed at the end of Subsec.IVA

\section{DIMENSIONAL ANALYSIS AND ENERGY BALANCE}

The model system contains three independent length scales: the diameter $a$ of a disk and the box sizes $L_{x}$ and $L_{y}$. In

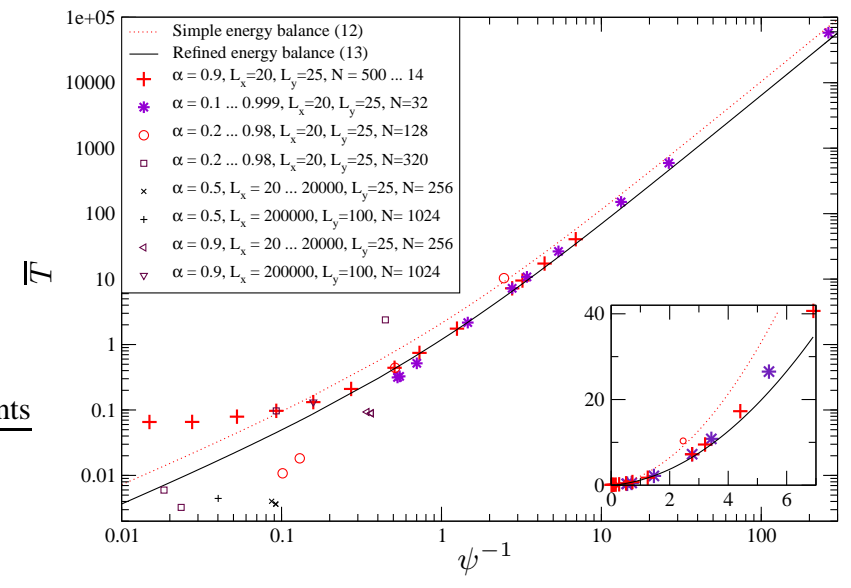

FIG. 2: Global granular temperature $\bar{T}$ as a function of the parameter $\psi^{-1}$ defined below (12). Comparison between simulations and the simple energy-balance argument, Eq. (12), as well as with the refined version, Eq. 13. The inset shows the same graph but on on a nonlogarithmic scale.

addition, there is one independent velocity scale, the driving velocity $v_{\text {drive }}$, and one independent mass scale, the mass of a disk $m$. Together with the initial positions and velocities of the particles that exhausts all dimensional quantities entering the time evolution of the system.

We would like to describe the system using dimensionless variables that do not depend on the initial conditions since we expect stationary states to be independent thereof. Therefore, we will measure all lengths in units of the particle diameter $a$, all times in units of $a / v_{\text {drive }}$ and all energies in

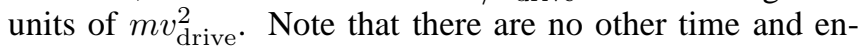
ergy scales. Thus, we introduce dimensionless variables: box sizes $\tilde{L}_{x}=L_{x} / a$ and $\tilde{L}_{y}=L_{y} / a$, granular temperatures $\tilde{T}_{x}=T_{x} /\left(m v_{\text {drive }}^{2}\right)$ and $\tilde{T}_{y}=T_{y} /\left(m v_{\text {drive }}^{2}\right)$, and the stress tensor $\tilde{\boldsymbol{\sigma}}=\boldsymbol{\sigma} a^{2} /\left(m v_{\text {drive }}^{2}\right)$. In the stationary state all dimensionless variables like $\tilde{T}$ and $\tilde{\boldsymbol{\sigma}}$, are independent of the driving velocity and only depend on position $\boldsymbol{r}$ and the remaining 4 independent dimensionless system parameters, which characterize the system completely: the number of disks $N$, the two edges of length of the system $\tilde{L}_{x}$ and $\tilde{L}_{y}$ in units of $a$, and the coefficient of normal restitution $\alpha$, which is a dimensionless material constant.

For simplicity of the notation we refrain from indicating dimensionless quantities by a tilde from now on. This should not cause confusion, because quantities having a physical dimension will not occur any more in the rest of the paper (except for the appendix).

We are interested in the macroscopic limit, which is taken such that $N \rightarrow \infty$ and $L_{y} \rightarrow \infty$ with a fixed line density $\lambda:=N / L_{y}$ of particles. Thus, in the macroscopic limit the number of system parameters is further reduced to $L_{x}, \lambda$, and $\alpha$. It is important to keep $L_{x}$ finite, otherwise energy balance would not work: energy input occurs only at boundaries, while energy dissipation is a bulk phenomenon.

It is instructive to estimate the average or global granular 
temperature $\bar{T}:=\int_{-L_{x} / 2}^{L_{x} / 2} d x \rho(x) T(x)$ by balancing the energy input at the walls and the energy loss due to particle collisions in the bulk [33, 63]. As shown in the appendix, we find that the granular temperature in the stationary state is independent of the initial data and given by

$$
\bar{T}=\left(\frac{2}{\pi}\right)^{3} \psi^{-2}\left(1+\sqrt{1+(\pi / 2)^{2} \psi}\right)^{2}
$$

where $\psi:=\sqrt{2} \chi \lambda\left(1-\alpha^{2}\right)$ and $\chi$ stands for the pair correlation at contact of the corresponding elastic system, which we estimate by the Henderson approximation (A.6. In Fig. 2 we plot the global granular temperature $\bar{T}$ as a function of $\psi^{-1}$ and compare it with simulations. For small values of $\psi^{-1}$ the simulation deviates significantly from this simple theory's prediction. For large values of $\psi^{-1}$ (dilute and/or quasi-elastic systems) the agreement is reasonable. In addition, we show the result

$$
\bar{T}=\frac{1}{2 \pi \psi^{2}}(1+\sqrt{1+\psi / 2})^{2}
$$

of a more refined calculation, which uses the pseudoLiouville-operator approach to kinetic theory and will be presented elsewhere. Eq. (13) yields a better agreement with the data for intermediate values of $\psi^{-1}$.

It is worth noting that the same type of argument which led to (12) also predicts that there is no generic stationary state for systems with the multiplicative driving mechanism A.1, described by $v_{\text {drive }}=0$ and a coefficient of restitution bigger than one for particle-wall collisions. Instead, such systems either cool down or heat up according to Haff's law [64], see Eq. A.9 in the appendix. This was also confirmed by simulations (not presented).

\section{HYDRODYNAMIC FIELDS IN SIMULATIONS}

In this section we discuss the results of our simulations for the hydrodynamic fields, as computed from Eqs. (6) - 111. Due to the absence of a local velocity field $\boldsymbol{V}$, these equations simplify accordingly. We have performed simulations for a wide range of system parameters $\alpha, \phi_{0}, L_{x}, L_{y}$, and present examples thereof below. We then go on in Sec. $\nabla$ to discuss the question of a local equation of state, relating $p(x), \rho(x)$, and $T(x)$.

\section{A. Density and temperature profiles}

We first present data for the density and temperature to demonstrate that the system is strongly inhomogeneous even for collisions with $\alpha=0.9$ and moderate densities. The parameters have been chosen as $L_{x}=20, L_{y}=25$, and $N=256$ so that the global area fraction is $\phi_{0}:=$ $\pi a^{2} N /(4 V)=0.4$. In Fig. 3 we show the local area fraction $\phi(x)$, the $x$ - and $y$-component of the granular temperature, $T_{x}(x)$ and $T_{y}(x)$, as well as the isotropic temperature $T(x)=\left[T_{x}(x)+T_{y}(x)\right] / 2$. We note that all quantities are

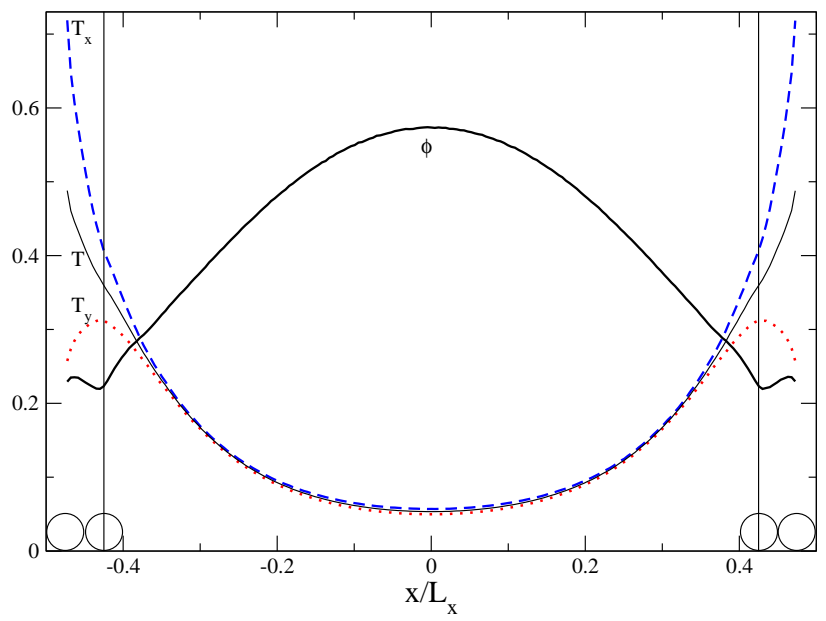

FIG. 3: Spatial profiles of the area fraction $\phi$ and granular temperatures $T_{x}, T_{y}$, and $T$ from simulations $\left[\alpha=0.9, L_{x}=20\right.$, $\lambda=10.24, N=256, \phi_{0}=0.4$, corresponding to a mean-free path $\left.\ell / L_{x} \approx 0.02\right]$.

symmetric in $x$ as expected. Except for a small area of approximately one disk diameter next to the walls (indicated by the vertical lines), the area fraction is a monotonic function for either half of the system with the maximal value in the middle of the system. The temperatures $T_{x}, T_{y}$, and $T$ are monotonic as well with the lowest temperatures in the middle of the sample.

The reason for the increased density next to the wall is an effective attractive potential of the wall due to entropic effects: Once a disks gets closer to the wall than one disk diameter, it can only receive hits from within the box but no hits from the direction of the wall. Thus the particle is pushed closer to the wall [65, 66]. This effect is partially compensated by the driving walls, which add momentum to any particle hitting the walls.

To support this explanation we have also investigated systems of half the size $-0.5 \leq x / L_{x} \leq 0$, half the number of particles and with an elastic wall at $x=0$. The resulting hydrodynamic fields (not shown) are almost identical to the ones in Fig. 3 except for a small region of about one diameter close to $x=0$ where the aforementioned effect is particularly visible.

In systems exhibiting stripe states, we have sometimes observed an oscillatory instability of a central dense cluster in the $x$-direction [62], in particular if $\alpha$ is low and $L_{x}$ is large. However, these oscillations occur on far shorter time scales compared to the time interval $\tau$, over which we average to obtain stationary-state quantities. Hence, these oscillations are completely averaged out in the presented data.

\section{B. Stress tensor and pressure}

In Figs. 4- 6 we show the components of the stress tensor $\sigma_{x x}(x), \sigma_{y y}(x)$, and $\sigma_{x y}(x)$ for two different sets of parame- 


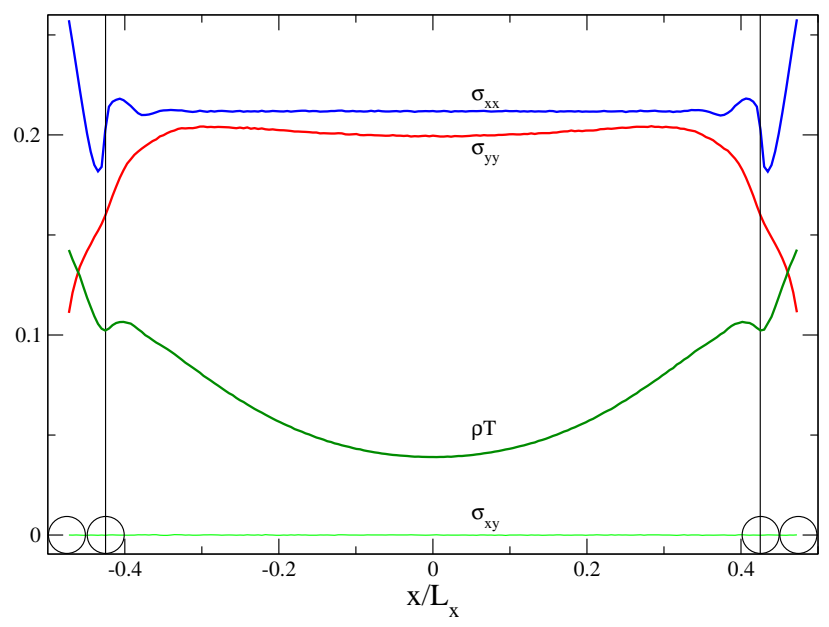

FIG. 4: Spatial profiles of the stress tensor components $\sigma_{x x}, \sigma_{y y}$, $\sigma_{x y}$, and the product $\rho T$ for the system of Fig. 3 For all $x$ away from the boundary layers, $\rho T$ is hardly to distinguish from $\rho T_{x}$ and $\rho T_{y}$ (not shown).

ters. Fig. (4) is typical for systems with quite elastic collisions $(\alpha=0.9)$ and moderate densities $\left(\phi_{0}=0.4\right)$, whereas Figs. [5] and 6 show rather dilute systems $\left(\phi_{0}=0.15\right)$ at two different inelasticities, a moderate one ( $\alpha=0.9$, as in Fig. (4) and a strong one $(\alpha=0.5)$. The off-diagonal component $\sigma_{x y}$ of the stress tensor is always vanishingly small. The $x x$-component $\sigma_{x x}$ is constant within the sample except for a boundary layer close to the driving walls, whereas Fig. 4 displays a dip of $\sigma_{y y}$ in the center of the sample, which is more pronounced for the more dilute system in Fig. 5 and hardly visible in the strongly inelastic, dilute system of Fig. 6 Furthermore, $\sigma_{y y}$ increases considerably over a broad range in Fig. 6 when moving from a driving wall towards the center of the system. Currently, the origin of both $x$-dependences of $\sigma_{y y}$ is not clear to us. While the stationary-state condition $\boldsymbol{\nabla} \cdot \boldsymbol{\sigma}=0$ and homogeneity in $y$ require $\sigma_{x x}$ and $\sigma_{x y}$ to be constant in $x$ on general grounds, this is not the case for $\sigma_{y y}$. We have carefully checked that the $x$-dependence of $\sigma_{y y}$ is not caused by a shear instability associated with a non-zero velocity field $\boldsymbol{V}(x)$ in the system. Thus, there are normal stresses present in the simulated systems, and they depend on $x$.

If the equation of state of the ideal gas held, the local pressure $p(x)$ would be related to the local temperature and density according to $p(x)=\rho(x) T(x)=(4 / \pi) \phi(x) T(x)$. For our system the kinetic part of the stress tensor is diagonal and each component is simply related to the corresponding temperature $\sigma_{x x}^{\mathrm{kin}}(x)=\rho(x) T_{x}(x)$ and $\sigma_{y y}^{\mathrm{kin}}(x)=\rho(x) T_{y}(x)$. Hence the difference between the measured stress tensor and the ideal gas behavior is due to collisions. From Figs. 4 - 6 it can be seen that the stress tensor deviates strongly from the ideal gas behavior. Consequently, collisions contribute significantly. Here the collisional part has been measured directly in the simulations and not only estimated by approximate theories as has been done elsewhere [30, 31].

We have also estimated the global mean free path $\ell$ for

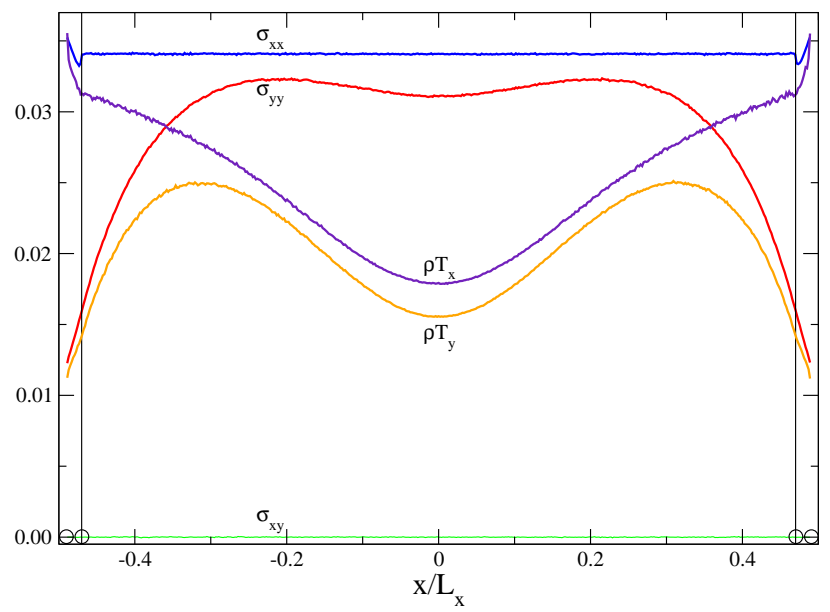

FIG. 5: Same as Fig. 4 but for a less dense system $\left[\alpha=0.9, L_{x}=\right.$ $\left.50, \lambda=9.6, N=240, \phi_{0}=0.15, \ell / L_{x} \approx 0.03\right]$.

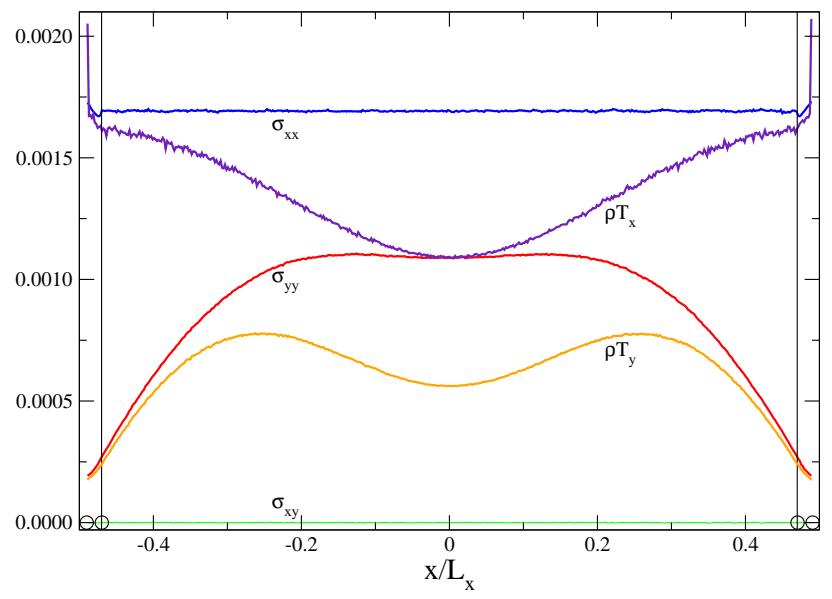

FIG. 6: Same as Fig. 5 but for $\alpha=0.5$.

our simulated systems according to Eq. (9) in [2], which expresses $\ell$ solely as a function of the global volume fraction $\phi_{0}$. For the denser system with $\phi_{0}=0.4$, shown in Figs. 3 and 4 this gives $\ell \approx 0.5$ (in units of the diameter of the disks, that is $\left.\ell / L_{x} \approx 0.025\right)$. For the thinner systems with $\phi_{0}=0.15$, shown in Figs. 5 and 6 one gets $\ell \approx 1.65$, that is $\ell / L_{x} \approx 0.033$. Thus, in all cases the mean free path $\ell$ is much smaller than the scales governing the spatial variations of the hydrodynamic fields, which are of order $L_{x}$.

\section{Density scaling}

For low global area fractions, $\phi_{0} \lesssim 0.01$, we observe scaling of the relative local area fraction $\phi(x) / \phi_{0}$ when plotted versus $x / L_{x}$. This is shown in Fig. 7 where we compare density profiles of systems with the same degree of inelasticity $\alpha=0.9$ and the same line density $\lambda=10.24$, but different values of the box width $1280 \leq L_{x} \leq 20000$, correspond- 


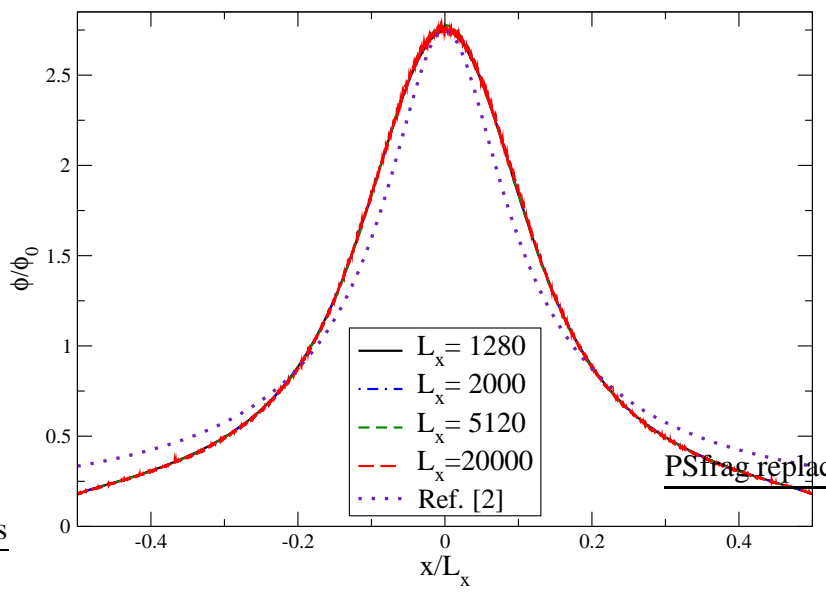

FIG. 7: Master curve for rescaled local area fraction in different lowdensity systems with the same line density $[\alpha=0.9, \lambda=10.24$, $\left.\ell / L_{x} \approx 0.03\right]$. The dotted line corresponds to the theoretical prediction of [2].

ing to global area fraction $\phi_{0}$ between $6 \cdot 10^{-3}$ and $4 \cdot 10^{-4}$. The relative local area fraction $\phi / \phi_{0}$ is seen to be a function of $x / L_{x}$ only and does not depend separately on $L_{x}$ and $\phi_{0}$. If the global area fraction were increased beyond $\phi_{0} \approx 0.01$ (not shown), then the data collapse would cease to hold. In addition, the height of the peak would be reduced and instead of the bell-shaped master curve in Fig.7 7 one would get overall concave profiles, similar to the one in Fig. 3

The hydrodynamic approach to quasi elastic, driven granular gases in Sec. II D in [2] predicts for low-density systems that the mean free path in units of $L_{x}$ depends only on the line density according to $\ell / L_{x}=1 /(\sqrt{8} \lambda)$. When computed for the situation of Fig. 7 one gets the small numerical value $\ell / L_{x} \approx 0.03$, indicating that $\ell$ is not a relevant length scale for the master curve of the rescaled spatial density profiles. The theory of [2] also makes a prediction for the relative local area fraction $\phi(x) / \phi_{0}$ in terms of the solution of a first-order differential equation. This differential equation includes one free parameter, which we have fitted in order to match the solution (dotted line in Fig. 7) at $x=0$ with the master curve from our simulations. The agreement is reasonable, showing that inelasticities of $\alpha=0.9$ are at the borderline of the scope of this otherwise powerful approach to quasi elastic, driven granular gases.

\section{LOCAL EQUATION OF STATE}

Having measured hydrodynamic fields in the steady state of the system, one is led to search for a relation among them. Such a constitutive equation is needed, for example, in all hydrodynamic approaches to driven granular gases in order to obtain a closed set of equations [2, 11, 30]. In the last section it was shown that a driven granular gas is intrinsically inhomogeneous. Therefore it is only natural to investigate how the local values of the granular temperature $T(x)$, pressure $p(x)$,

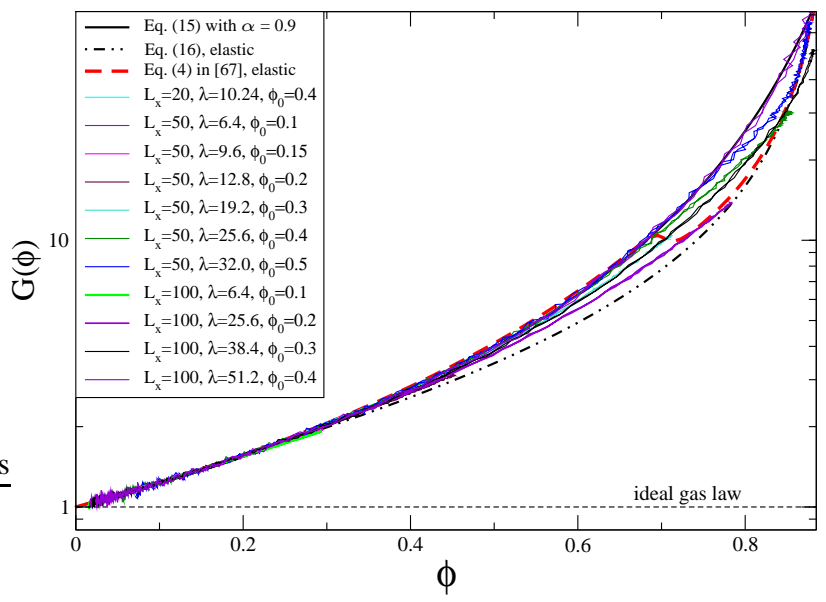

FIG. 8: (Color online) Semilogarithmic parametric plots of the function $G(\phi)$ from the local equation of state 14 for different systems $\left[\alpha=0.9, L_{y}=25,0.01 \leq \ell / L_{x} \leq 0.05\right]$. Also shown are the theoretical predictions from [15, [16, and Eq. (4) in [67].

and density $\rho(x)$-resp. area fraction $\phi(x)=\rho(x) \pi / 4$ in our units - are related to each other. To do so we observe in Fig. 3 that $T(x), p(x)$, and $\phi(x)$ are all symmetric in $x$. Moreover, and this is crucial, $\phi(x)$ is monotone in $x$ for either sign of $x$ (except for a boundary layer of approximately one diameter in width close to a driving wall, which we ignore). Therefore one can invert the function $\phi(x)$ for positive $x$. Upon inserting $x=\phi^{-1}(\phi(x))$ into the local pressure and temperature, we arrive at the constitutive equation

$$
\frac{p(x)}{\rho(x) T(x)}=G(\phi(x))
$$

with some function $G$. Fig. 8 shows parametric plots on a semilogarithmic scale of the function $G$ with the values of $p(x) /[\rho(x) T(x)]$ plotted against those of $\phi(x)$ for all $x$ (except for those in the boundary layer mentioned above). In order to utilize a broad range of $\phi$-values, Fig. 8 contains data from 11 different systems, all of which have the same coefficient of restitution $\alpha=0.9$ and the same height $L_{y}=25$, but different widths $L_{x}$ and different global area fractions $\phi_{0}$. For not too large values of $\phi$ these data merge quite nicely, indicating that there is only a weak dependence of $G$ on the global system parameters $L_{x}$ and $\phi_{0}$ in the corresponding parameter range. In this case the constitutive equation (14) can be interpreted as the local equation of state of the system. The horizontal line in Fig. 8 marks ideal-gas behavior, from which $G$ deviates due to the collisional contribution to the pressure. These deviations increase significantly with increasing $\phi$.

The dotted line in Fig. 8 corresponds to the function

$$
G(\phi)=1+(1+\alpha) \phi \chi,
$$

where $\chi$ stands for the pair correlation function at contact of the associated elastic hard-sphere gas in thermal equilibrium [73]. Since $\chi$ is not known exactly, we estimate it by the Henderson approximation A.6 for numerical purposes. In the 
context of granular gases, (15) occurred originally in a global equation of state for the homogeneous situation of a freely cooling granular gas [1]. In [68] the pair correlation function has been studied in a homogeneously driven inelastic system with periodic boundary conditions. It was found to be nearly independent of the coefficient of restitution and well approximated by the Henderson approximation, Eq. A.6. In [31] this form of $G$ was used in the local equation of state (14) to get a theoretical prediction for $p(x)$ from simulations of $T(x)$ and $\phi(x)$ in a driven granular gas. Fig. 8 reveals that this works generally quite well for up to rather high local area fractions $\phi(x) \lesssim 0.5$. In even denser systems agreement still holds for the well fluidized parts. Deviations from (15) start to occur when entering the transition zones to the frozen-out stripe of particles in the center of these high-density systems. The dashed-dotted line in Fig. 8 corresponds to the interpolation formula [2]

$$
G(\phi)=\frac{\phi_{c}+\phi}{\phi_{c}-\phi}
$$

for $0 \leq \phi<\phi_{c}$, which connects the behavior of dilute (van der Waals) and dense (ordered) elastic hard-sphere systems. Here $\phi_{c}:=\pi /(2 \sqrt{3}) \approx 0.91$ denotes the area fraction for ordered closed packings in two dimensions. Eq. (16) was applied to quasi-elastic granular gases in [2], and even for our simulations with $\alpha=0.9$ there is agreement with the local data in the low-density regions up to $\phi(x) \lesssim 0.4$. In addition, the most dense regions of regularly ordered, frozen-out particles in the center of the high-density systems are described correctly, too. Yet another interpolation formula for $G$, which is rather accurate for an elastic hard-sphere gas even in the vicinity of the freezing transition, was put forward in Eq. (4) in [67] and is depicted by the dashed line in Fig. [8 Thus, the crossover between fluidized and frozen-out behavior in the inelastic, driven systems, originating from the transition zone at the border of the solidified stripe of particles in the system center, is very smooth in comparison to the coexistence region of the freezing transition for an equilibrated elastic gas. Moreover, the location of the crossover depends on the global system parameters. Since both interpolation formulae, (16) and the one from [67], were tailored for elastic systems in thermal equilibrium, it is surprising to find such a good agreement with the local data for driven inelastic hard-sphere gases with restitution $\alpha=0.9$.

When lowering the coefficient of restitution $\alpha$ from 0.9 to 0.8 (not shown), there are only little changes to the plot in Fig. 8 In particular, the spread of the data pertaining to different systems increases, even for low densities. Still one may be inclined to interpret (14) as a local equation of state-at least approximately. This situation is intermediate to the previous with $\alpha=0.9$ and the following for strongly inelastic systems with coefficient of restitution $\alpha=0.5$. Indeed, Fig. 9 reveals major discrepancies in the local pressure, which are due to the systems with global area fractions above $\phi_{0} \approx 0.2$. The discrepancies occur even at positions in the sample where the local area fractions are below 0.2 and where all the more dilute systems, i.e. those with $\phi_{0} \leq 0.2$, agree reasonably with the proposal (15). The concept of a local equation of state is

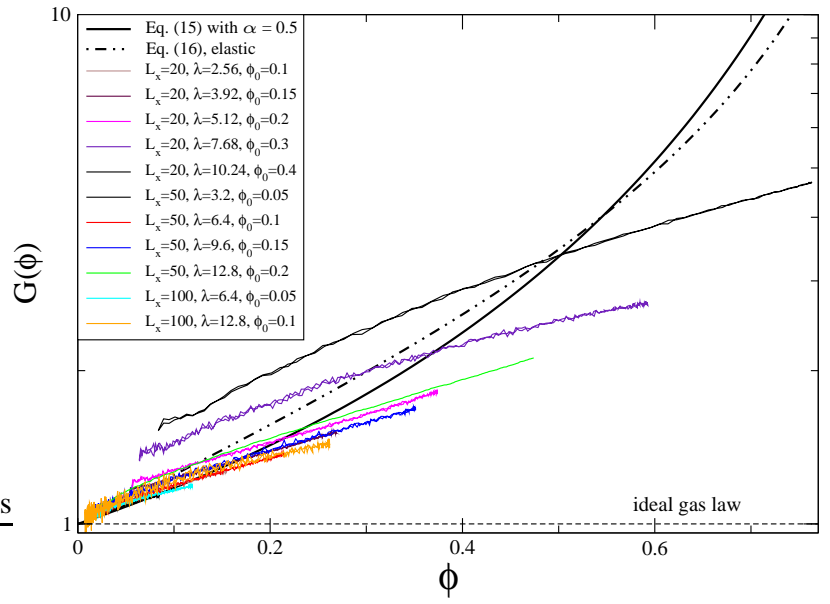

FIG. 9: (Color online) Same as Fig. 8 but for more inelastic systems $\left[\alpha=0.5, L_{y}=25,0.01 \leq \ell / L_{x} \leq 0.1\right]$.

therefore not sustainable any more for such strongly inelastic systems, and (14) merely plays the role of a local constitutive equation, which depends in addition on the global system parameters.

\section{ABSENCE OF SCALING FOR VELOCITY DISTRIBUTIONS}

Finally, we examine the local velocity distributions of the driven granular gas in the stationary state. We distinguish between the local distribution

$$
f_{x}\left(x, v_{x}\right):=\frac{1}{\rho(x)} \int_{\mathbb{R}} d v_{y} f_{\text {stat }}\left(x, v_{x}, v_{y}\right)
$$

at position $x$ of the velocity component $v_{x}$ in the direction of the driving and the distribution

$$
f_{y}\left(x, v_{y}\right):=\frac{1}{\rho(x)} \int_{\mathbb{R}} d v_{x} f_{\text {stat }}\left(x, v_{x}, v_{y}\right)
$$

of the velocity component $v_{y}$ perpendicular to the direction of the driving. By definition, these velocity distributions are normalized to unity.

In order to determine $f_{x}$ and $f_{y}$ from the simulation we use two different methods. The first one extracts them directly according to their definitions (17) and (18) from $f_{\text {stat }}$, which is determined as described in Subsec. [IC One disadvantage of this method is that it yields a smeared-out velocity distribution, which is spatially averaged over the width of the strip $\mathcal{V}_{x}$ centered around $x$. This is of practically no importance in the middle of the simulation box, but strongly disturbing for resolving the subtleties which occur close to the driving walls and are presented in Subsec. VIA The second way of measuring the velocity distributions avoids this problem: To get $f_{i}$ ( $i=x$ or $y$ ) we keep track of all those particles which pass the line parallel to the $y$-axis at position $x$ within a very long time interval of length $\tau$ and whose $i$ th component of the velocity 
lies in a small interval of width $\Delta v$ around $v_{i}$. Let us enumerate these particles by $n_{i}$ and denote their velocity components by $v_{x}^{\left(n_{i}\right)}$ and $v_{y}^{\left(n_{i}\right)}$. Then one has (in the limits $\tau \rightarrow \infty$ and $\Delta v \rightarrow 0)$

$$
\rho(x) f_{i}\left(x, v_{i}\right)=\frac{1}{\tau \Delta v L_{y}} \sum_{n_{i}} \frac{1}{\left|v_{x}^{\left(n_{i}\right)}\right|},
$$

because for $i=x$, resp. $i=y$, the right-hand side of 19 is equal to

$$
\int_{\mathbb{R}} d v_{y} \frac{\left|F_{x}\left(x, v_{x}, v_{y}\right)\right|}{\left|v_{x}\right|} \quad \text { resp. } \int_{\mathbb{R}} d v_{x} \frac{\left|F_{x}\left(x, v_{x}, v_{y}\right)\right|}{\left|v_{x}\right|} .
$$

Here, $F_{x}\left(x, v_{x}, v_{y}\right):=v_{x} f_{\text {stat }}\left(x, v_{x}, v_{y}\right)$ denotes the $x$ component of the (differential) current density in the stationary state at position $x$ of particles with velocity components $v_{x}$ and $v_{y}$.

As compared to the first method of measuring velocity distributions, this one is also statistically more effective for determining rare events, such as the high-velocity tails in Subsec.VIB However, as far as $f_{x}\left(x, v_{x}\right)$ is concerned for small $v_{x}$, the second method is inferior to the first, because (19) assigns a large weight to the relevant events and therefore amplifies statistical fluctuations, too.

\section{A. Effects of the discontinuity at a driving wall}

Particle-number conservation at a driving wall requires the incoming particle flux at the wall to be equal to the outgoing flux. For the velocity distribution $f_{x}$ this implies the boundary condition [30]

$$
\begin{aligned}
f_{x}\left(\mp L_{x} / 2, v_{x}\right)= & \Theta\left( \pm v_{x}-v_{\text {drive }}\right)\left(1 \mp \frac{v_{\text {drive }}}{v_{x}}\right) \\
& \times f_{x}\left(\mp L_{x} / 2,-v_{x}+v_{\text {drive }}\right),
\end{aligned}
$$

which must hold for all $v_{x}>0$. Here $\Theta(z):=1$ for $z \geq 0$, respectively $\Theta(z):=0$ for $z<0$, denotes Heaviside's unit-step function and $v_{\text {drive }}=1$ in our units chosen. The boundary condition (21) relates the distribution $f_{x}$ of velocities prior to a collision with the wall to the one after a collision with the wall. In contrast, the velocity distribution $f_{y}$ must obey the usual reflection symmetry in $v_{y}$ everywhere in the system, that is

$$
f_{y}\left(x, v_{y}\right)=f_{y}\left(x,-v_{y}\right)
$$

for all $|x| \leq L_{x}$ and all $v_{y}>0$.

We measured the velocity distribution $f_{x}\left(x, v_{x}\right)$ at 25 different positions in a moderately inelastic system with coefficient of restitution $\alpha=0.9$. Fig. 10 shows the rescaled velocity distribution $\tilde{f}_{x}\left(x, v_{x} / \sqrt{T_{x}(x)}\right):=f_{x}\left(x, v_{x}\right) \sqrt{T_{x}(x)}$, measured using method one. At the driving walls $\tilde{f}_{x}$ is seen to obey the boundary condition 21. When moving from a driving wall towards the center of the system, the gap in $\tilde{f}_{x}$ gets gradually smeared out, and $\tilde{f}_{x}$ becomes more and more

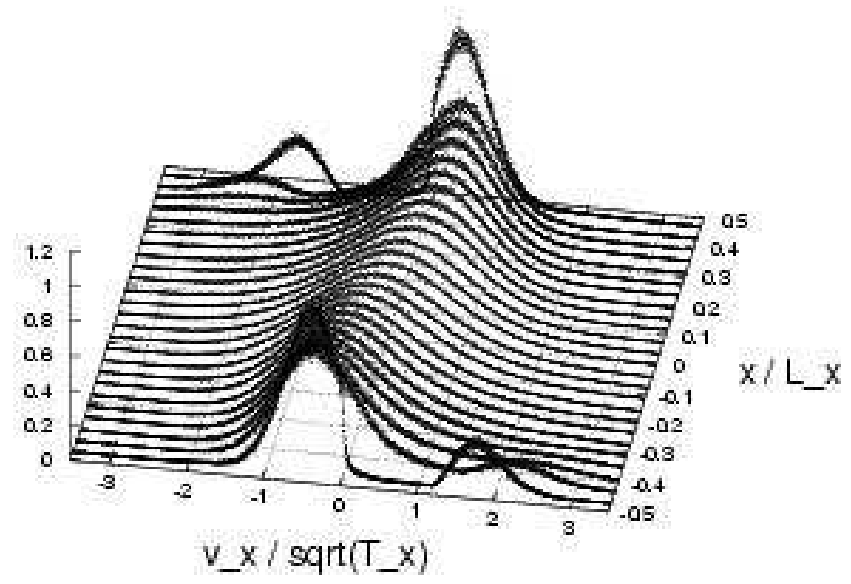

FIG. 10: Rescaled velocity distributions $\tilde{f}_{x}$ for different $x$. [System parameters: $\left.\alpha=0.9, L_{x}=20, \lambda=10.24\left(N=256, \phi_{0}=0.4\right)\right]$

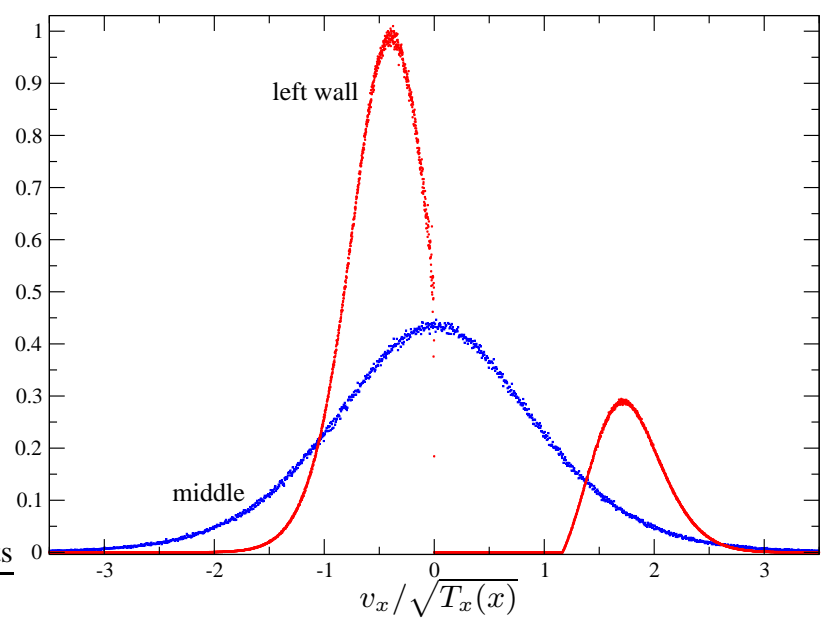

FIG. 11: Two rescaled velocity distributions $\tilde{f}_{x}$ from Fig. 10 at the left wall and in the middle of the system.

symmetric. Clearly, even for this moderately inelastic system $f_{x}$ does not scale for different $x$. The two extreme cases for $x=-L_{x} / 2$ and $x=0$ are shown together in Fig. 11 The data for $\tilde{f}_{x}$ at the wall was obtained with the second method for measuring velocity distributions. This result is in agreement with Direct-Simulation-Monte-Carlo results in [30] and Molecular-Dynamics simulations in [31].

\section{B. High-velocity tails}

Our main result for the velocity distributions is the nonscaling and multiformity of their tails. In order to observe these phenomena, extensive simulations for capturing rare events are required and the data have to be analyzed on a logarithmic scale. In contrast, on a linear scale the rescaled velocity distributions seem to collapse approximately, as was ob- 


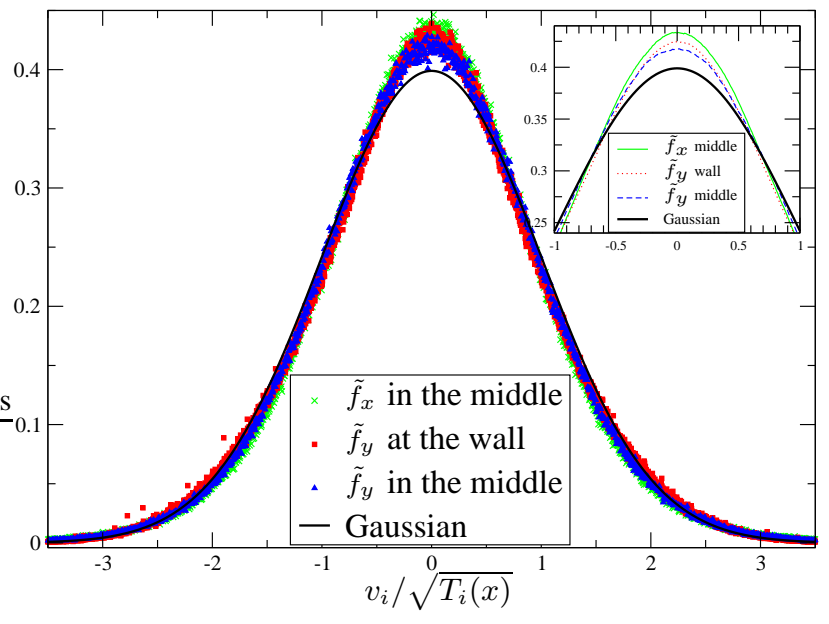

FIG. 12: (Color online) Combined plot of rescaled velocity distributions of the same system as in Figs. 10 and $11 \tilde{f}_{x}$ in the middle of the sample (green crosses), $\tilde{f}_{y}$ at a driving wall (red squares), and $\tilde{f}_{y}$ in the middle (blue triangles). Also shown is a centered Gaussian with unit variance (black solid line). The inset shows the central part of the main graph with the (1433/2964/1340) data points being smoothed (running average over (41/85/38) data points).

served previously by e.g. [2, 69]. As an example we show in Fig. 12 the rescaled distributions $\tilde{f}_{y}\left(x, v_{y} / \sqrt{T_{y}(x)}\right):=$ $f_{y}\left(x, v_{y}\right) \sqrt{T_{y}(x)}$ in the middle of the sample $(x=0)$ and at a driving wall $\left(x= \pm L_{x} / 2\right)$ for the same moderately inelastic system $(\alpha=0.9)$ as in Figs. 10 and 11 Clearly, the curves are reflection-symmetric in $v_{y}$. For comparison, we have also included the symmetric distribution $\tilde{f}_{x}$ in the middle of the system. Deviations are visible for small velocities only, as the inset of Fig. 12 shows. The corresponding Gaussian (solid line) in Fig. 12 suggests that the velocity distributions are close to but not identical to a Maxwellian. The approximate data collapse, observed on this level of accuracy in Fig. 12. even continues to hold if the coefficient of restitution of the gas is varied in a not too large extent. In considerably more inelastic systems, such as for $\alpha=0.5$, this is not true any longer. For example, the peak of $\tilde{f}_{x}$ measured in the middle of the system would be considerably broader and flatter than the ones of $\tilde{f}_{y}$ in the center and at the wall (not shown).

In contrast, Fig. 13 a) shows the same data of Fig. 12 on a semi-logarithmic scale and includes also velocities of much higher absolute values. From this figure it is evident that scaling does not hold in the high-velocity tails of the distributions either. Similar observations were made before in e.g. [31, 45, 46]. The type of decay in the high-velocity tails is different for $\tilde{f}_{x}$ and $\tilde{f}_{y}$, and also depends on the position in the sample, the coefficient of restitution $\alpha$, and the global area fraction $\phi_{0}$. This is illustrated by examples of different systems in Fig. 13 In the insets we show $\left|\ln \tilde{f}_{i}\right|^{-1}$ on a doublelogarithmic scale in order to determine the decay exponent $\beta$ defined by

$$
\ln \tilde{f}_{y}\left(0, v_{y} / \sqrt{T_{y}(0)}\right) \stackrel{\left|v_{y}\right| \rightarrow \infty}{\sim}-\left|v_{y} / \sqrt{T_{y}(0)}\right|^{\beta}
$$

for $\tilde{f}_{y}$ in the middle of the sample. The exponent is defined accordingly for $\tilde{f}_{y}$ at the wall and $\tilde{f}_{x}$ in the middle of the sample. For the moderately inelastic systems with $\alpha=0.9$ in the first row of Fig. 13 the asymptotics has been clearly reached. We note that $\beta$ is different for the different distributions, and also depends on the global area fraction $\phi_{0}$. Upon lowering $\alpha$ (top to bottom in Fig. 13) and/or decreasing the global area fraction $\phi_{0}$ (left to right), the tails of $\tilde{f}_{y}$ get more and more populated, that is, $\beta$ decreases. In very simple terms, this may be understood from the fact that (i) the largest typical velocities are always of the order of $v_{\text {drive }}=1$ (see also Fig. 114) and (ii) that $v_{\text {drive }} / \sqrt{T_{i}(x)}$ increases up to 20 with decreasing $\alpha$ and decreasing $\phi_{0}$. Hence, a Maxwellian velocity distribution would not be able to supply enough probability to particles with velocities of the order of $v_{\text {drive }}$, instead higher-populated tails are needed. This argument suggests different behavior in different velocity regions so that the distributions cannot be fitted to the functional form (23) over the entire range of velocities [45]. Indeed, such a behavior can be seen in Fig. 13] d), e) and f). The final asymptotics could not always be deduced from the simulations, even though our data include velocities which are up to 40 times bigger than the appropriate granular velocities $\sqrt{T_{i}}$. This applies to $\tilde{f}_{y}$ in the middle of the sample in part e), where we suspect that the final asymptotics has not been reached. An asymptotic analysis of $\tilde{f}_{x}$ in the middle of the sample is even more problematic due to particles that reach the system center from a driving wall without undergoing a collision. These particles give rise to the side peaks of $\tilde{f}_{x}$ in parts e) and f), which have prevented us from determining $\beta$ in these cases.

Fig. 13 contains smoothed data as in the inset of Fig.12 and the scales of the horizontal axes are determined by the square root of the appropriate granular temperatures. For comparison, Fig. 14 shows the unsmoothed data corresponding to Fig. 13f f), plotted directly versus $v_{i}$ (in units of $v_{\text {drive }}$ ). The side peaks of $\tilde{f}_{x}$ in the middle of the sample are due to the above-mentioned particles which fly from a driving wall to the system center without undergoing a collision. For the vast majority of particles $\left|v_{y}\right|$ is less than $80 \%$ of the driving velocity. It is in the region of this highest velocity observed for $\tilde{f}_{y}$ in the middle of the sample, where $\tilde{f}_{x}$ changes abruptly its slope.

The exponent $\beta$ has also been determined experimentally in a strongly driven gas so that gravity effects are small [16]. A value $\beta \approx 1.55$ was measured for a gas with coefficient of restitution $\alpha \approx 0.93$. It was found to be remarkably independent of the global area fraction $\phi_{0}$, which was varied from 0.05 to 0.3 . We have also simulated the system of [16] with zero gravity (not shown) and reproduced their value for $\beta$. Even though the experimental data cover a wide range of velocities, some of the interesting phenomena discussed in Fig. 13 cannot be observed in this range. For the same reason the significance of the particular value $\beta \approx 1.55$ should not be overestimated.

Simulations of a driven granular gas in a circularly shaped box also yield stretched Gaussian tails [45]. In addition, evidence is given that $\beta$ depends only on the coefficient of restitution and the average ratio of the number of particle-wall 

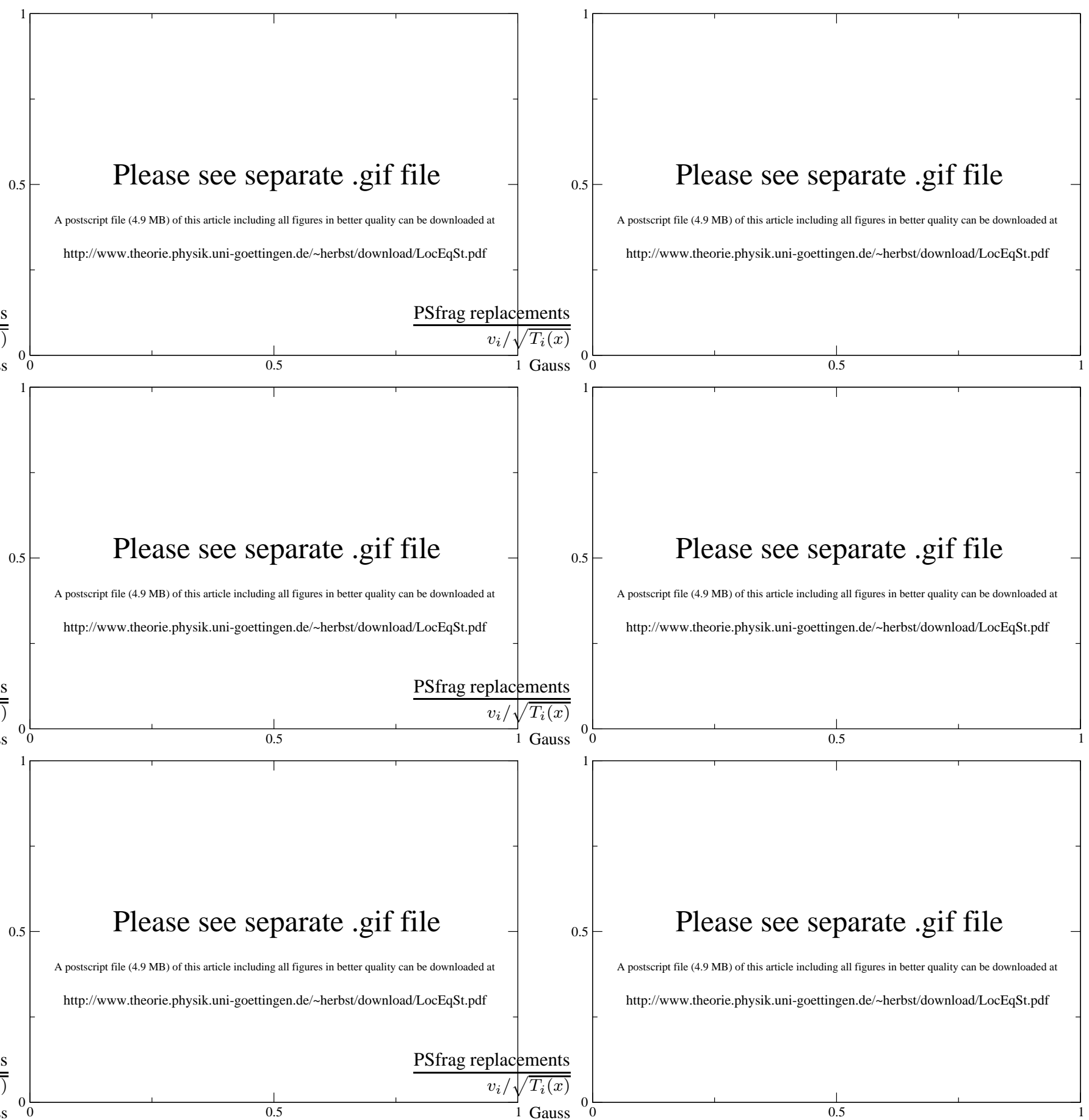

FIG. 13: (Color online) Rescaled velocity distributions $\tilde{f}_{x}$ in the middle of the sample (green crosses), $\tilde{f}_{y}$ at a driving wall (red squares), and $\tilde{f}_{y}$ in the middle (blue triangles) as in Fig. 12 but here smoothed data are shown on a semi-logarithmic scale and velocities of much higher absolute values are included. The solid line is a centered Gaussian with unit variance. Different parts of the figure represent different systems with decreasing global area fraction $\phi_{0}$ (left to right) and decreasing coefficient of restitution $\alpha$ (top to bottom). The remaining system data are $L_{x}=20, \lambda=10.24(N=256)$ for the left column and $L_{x}=50, \lambda=9.6(N=240)$ for the right column. The insets show $\left|\ln \tilde{f}_{i}\right|^{-1}$ on a double-logarithmic scale to determine the decay exponent $\beta$ from [23. The dashed line is the best linear fit to $\tilde{f}_{x}$ in the middle of the sample, the solid line to $\tilde{f}_{y}$ in the middle, and the dotted line to $\tilde{f}_{y}$ at the wall. Part a) corresponds to the system of Fig. 12 


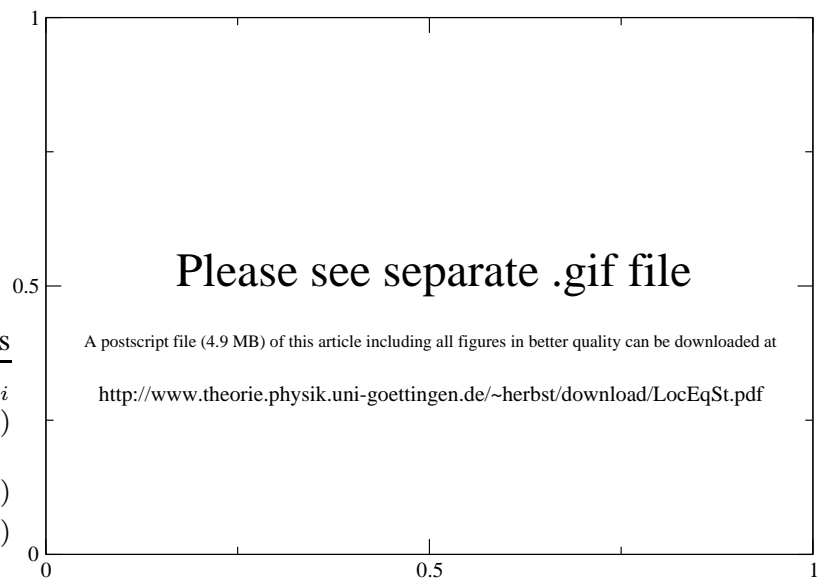

FIG. 14: (Color online) Velocity distributions for the system in Fig. 13 f), but with unsmoothed data and without rescaling of the horizontal axis.

collisions and particle-particle collisions. For homogeneously driven systems the tail of the velocity distribution was theoretically predicted [23] to be governed by the decay exponent $\beta=3 / 2$. Simulations of homogeneously driven systems [70, 71] observed the exponent $\beta=3 / 2$ only for unrealistically low values of the coefficient of restitution.

\section{SUMMARY AND OUTLOOK}

In this article we explored the steady-state properties of a granular gas driven by vibrating walls. We have measured the full stress tensor, including the collisional contribution. This allowed us to obtain a constitutive equation directly from the simulations. The constitutive equation relates local density, pressure and temperature. For small inelasticities it can be regarded as the local equation of state of the gas, because it is to a large extent independent of the global system parameters. For strongly inelastic systems this interpretation cannot be sustained, instead the constitutive relation depends on the global volume fraction and sample geometry. We have also measured local velocity distributions, whose high-velocity tails were found to depend on the position in the sample as well as on the coefficient of restitution and the global volume fraction. Moreover, the tails are different for the two velocity components parallel and perpendicular to the driving walls. To conclude, the stationary state of a driven granular gas is in general non-universal - in contrast to the corresponding elastic system. This is not unexpected because the driven granular gas is a non-equilibrium state. Furthermore driving the system by energy input through the walls is effective only if the distance between the driving walls is finite so that the sample geometry enters naturally.

We plan to extend our studies in various directions. It is straightforward to also consider rotational motion of the disks, by generalizing the collision rules to include tangential restitution as well as Coulomb friction. Again, the question arises to what extent the additional parameters affect the local equation of state. In this context it would be interesting to study the effects of gravity for better comparison with experiments. Another interesting quantity to measure is the heat flux which, like the stress tensor, is also expected to be affected by collisions. Finally, one might also investigate other driving mechanisms, like vibrations with finite frequency and non-zero amplitude or sinusoidal driving. Work along these lines is in progress.

\section{Acknowledgments}

We are grateful to Timo Aspelmeier for providing us with the first version of our program. Olaf Herbst thanks Stefan Luding and Isaac Goldhirsch for inspiring discussions. Finally, we acknowledge financial support by the DFG through SFB 602 and Grant No. Zi 209/6-1.

\section{APPENDIX: A SIMPLE ENERGY-BALANCE ARGUMENT}

Here we derive the energy balance Eq. (12) by using arguments as in [33, 63]. We do this in a slightly more general setting than needed for Eq. (12) and allow in addition for inelastic collisions with the wall, characterized by a coefficient of restitution $\alpha_{\mathrm{w}}$. The appropriate generalization of the collision rule (4) includes both the driving velocity and the coefficient of restitution with the wall,

$\boldsymbol{v}^{\prime}=\boldsymbol{v}+\Delta \boldsymbol{v}_{\mathrm{pw}} \quad$ with $\quad \Delta \boldsymbol{v}_{\mathrm{pw}}=\left[-\left(1+\alpha_{\mathrm{w}}\right) v_{x} \pm v_{\text {drive }}\right] \boldsymbol{e}_{x}$.

The special case $v_{\text {drive }}=0$ and $\alpha_{\mathrm{w}}>1$ provides an alternative driving mechanism, which, however, does not give rise to a stationary state, as will be shown below. In order to be able to treat this limit and for a better readability of the formulae, we refrain from using dimensionless physical units in this appendix.

The average energy gain $\Delta E_{\mathrm{pw}}$ due to a particle-wall collision is estimated from Eq. A.1 by averaging the kinetic energy before and after the collision with a Maxwellian velocity distribution with (global) temperature $T$. This gives

$$
\Delta E_{\mathrm{pw}}=\frac{m}{2}\left(v_{\text {drive }}^{2}+4 \alpha_{\mathrm{w}} \sqrt{\frac{T}{2 \pi m}} v_{\text {drive }}-\left(1-\alpha_{\mathrm{w}}^{2}\right) \frac{T}{m}\right) .
$$

The collision frequency of particles with the left (right) wall is estimated by

$$
f_{\mathrm{pw}}=\frac{N}{L_{x}} \sqrt{\frac{T}{2 \pi m}},
$$

where we have assumed the density to be spatially homogeneous throughout the system.

When two disks collide in the bulk, the average change in total energy is computed similarly to (A.2) from (2) and (3) by averaging pre- and post-collisional kinetic energy, which yields

$$
\Delta E_{\mathrm{pp}}=-\frac{1-\alpha^{2}}{2} T
$$


Finally, the number of particle-particle collisions per time is given approximately by Enskog's collision frequency [23]

$$
f_{\mathrm{pp}}=\frac{N}{L_{x}} \lambda a \chi \sqrt{\frac{T \pi}{m}},
$$

where $\chi$ is the pair correlation function at contact of a corresponding elastic gas in thermal equilibrium. Since $\chi$ is not exactly known, we resort to the widely used Henderson approximation [72]

$$
\chi \approx \frac{1-7 \phi / 16}{(1-\phi)^{2}}
$$

for numerical purposes. It may be viewed as a heuristic rational approximation to the virial expansion of $\chi$ and is the two-dimensional equivalent to the Carnahan-Starling approximation [66] for a three-dimensional hard-sphere gas. Additional higher-order terms to the Henderson approximation, which are proportional to $\phi^{3} /(1-\phi)^{4}$, have turned out to be irrelevant for our purposes and will therefore not be taken into account.

Summing over energy loss in the bulk and energy gain at the right and left wall, we obtain for the total change in granular temperature

$$
\begin{aligned}
\frac{d T}{d t} & \approx \frac{2 f_{\mathrm{pw}} E_{\mathrm{pw}}+f_{\mathrm{pp}} E_{\mathrm{pp}}}{N} \\
& =\frac{m}{L_{x}} \sqrt{\frac{T}{2 \pi m}}\left(v_{\text {drive }}^{2}+4 \alpha_{\mathrm{w}} \sqrt{\frac{T}{2 \pi m}} v_{\text {drive }}-\frac{\pi \psi_{\mathrm{eff}} T}{2 m}\right) .
\end{aligned}
$$

Here the dimensionless parameter

$$
\psi_{\mathrm{eff}}:=\psi-\frac{2}{\pi}\left(\alpha_{\mathrm{w}}^{2}-1\right)
$$

is given in terms of $\psi:=\sqrt{2} \lambda a \chi\left(1-\alpha^{2}\right)$, which was already introduced below Eq. (12). (We note that $\lambda a$ is the dimensionless line density employed there.) We briefly discuss two special cases:

a) For $v_{\text {drive }}=0$ and $\alpha_{\mathrm{w}}>1$ no stationary state is reached in general. Both, energy gain and loss increase like $T^{3 / 2}$, resulting in Haff's law

$$
\frac{d T}{d t}=-\frac{\psi_{\text {eff }}}{2 L_{x}} \sqrt{\frac{\pi}{2 m}} T^{3 / 2}
$$

which has been discussed extensively in the different context of freely cooling granular gases. Here the temperature continues to decrease or increase depending on whether dissipation or driving wins.

b) For $\alpha_{\mathrm{w}}=1$ and $v_{\text {drive }}>0$ the granular temperature adjusts to the driving so that the stationary state with $d T / d t=$ 0 is characterized by the quadratic equation

$$
1+2 \sqrt{\frac{2 \bar{T}}{\pi}}-\frac{\pi \psi}{2} \bar{T}=0
$$

The solution of A.10 for the dimensionless global temperature $\bar{T}:=T /\left(m v_{\text {drive }}^{2}\right)$ is given in Eq. (12).
[1] A. Goldshtein and M. Shapiro, J. Fluid Mech. 282, 75 (1995).

[2] E. L. Grossman, T. Zhou, and E. Ben-Naim, Phys. Rev. E 55, 4200 (1997).

[3] J. J. Brey, J. W. Dufty, C. S. Kim, and A. Santos, Phys. Rev. E 58, 4638 (1998).

[4] N. Sela and I. Goldhirsch, J. Fluid Mech. 361, 41 (1998).

[5] I. Goldhirsch, Annu. Rev. Fluid Mech. 35, 267 (2003).

[6] Y. Du, H. Li, and L. P. Kadanoff, Phys. Rev. Lett. 74, 1268 (1995).

[7] M.-L. Tan and I. Goldhirsch, Phys. Rev. Lett. 81, 3022 (1998).

[8] L. P. Kadanoff, Rev. Mod. Phys. 71, 435 (1999).

[9] H. M. Jaeger, S. R. Nagel, and R. P. Behringer, Rev. Mod. Phys. 68, 1259 (1996).

[10] E. Livne, B. Meerson, and P. V. Sasorov, Phys. Rev. E 66, 050301 (2002).

[11] E. Livne, B. Meerson, and P. V. Sasorov, Phys. Rev. E 65, 021302 (2002)

[12] B. Meerson, T. Pöschel, P. Sasorov, and T. Schwager, e-print cond-mat/0208286 to appear in Phys. Rev. E (2004).

[13] J. S. Olafsen and J. S. Urbach, Phys. Rev. Lett. 81, 4369 (1998).

[14] J. S. Urbach and J. S. Olafsen, in Granular Gases, edited by T. Pöschel and S. Luding (Springer, Berlin, 2001), Lecture Notes in Physics 564, pp. 410-428.

[15] W. Losert, D. G. W. Cooper, and J. P. Gollub, Phys. Rev. E 59, 5855 (1999).
[16] F. Rouyer and N. Menon, Phys. Rev. Lett. 85, 3676 (2000).

[17] A. Kudrolli, M. Wolpert, and J. P. Gollub, Phys. Rev. Lett. 78, 1383 (1997).

[18] E. Falcon, S. Fauve, and C. Laroche, Eur. Phys. J. B 9, 183 (1999).

[19] E. Falcon, R. Wunenburger, P. Evesque, S. Fauve, C. Chabot, Y. Garrabos, and D. Beysens, Phys. Rev. Lett. 83, 440 (1999).

[20] S. Warr, J. M. Huntley, and G. T. H. Jacques, Phys. Rev. E 52, 5583 (1995).

[21] S. F. E. Falcon and C. Laroche, in Granular Gases, edited by T. Pöschel and S. Luding (Springer, Berlin, 2000), Lecture Notes in Physics 564, pp. 244-253.

[22] D. R. M. Williams and F. C. MacKintosh, Phys. Rev. E 54, R9 (1996).

[23] T. P. C. van Noije and M. H. Ernst, Granular Matter 1, 57 (1998).

[24] T. P. C. van Noije, M. H. Ernst, E. Trizac, and I. Pagonabarraga, Phys. Rev. E 59, 4326 (1999).

[25] A. Barrat, T. Biben, Z. Racz, E. Trizac, and F. van Wijland, J. Phys. A: Math. Gen. 66, 463 (2002).

[26] A. Barrat, E. Trizac, and J. N. Fuchs, Eur. Phys. J. E 5, 161 (2001).

[27] R. Cafiero, S. Luding, and H. J. Herrmann, Phys. Rev. Lett. 84, 6014 (2000).

[28] J. J. Brey and D. Cubero, Phys. Rev. E 57, 2019 (1998). 
[29] D. Risso and P. Cordero, Phys. Rev. E 65, 021304 (2002).

[30] J. J. Brey, M. J. Ruiz-Montero, and F. Moreno, Phys. Rev. E 62, 5339 (2000).

[31] A. Barrat and E. Trizac, Phys. Rev. E 66, 051303 (2002).

[32] B. Drossel and T. Prellberg, Eur. Phys. J. B 1, 533 (1998).

[33] V. Kumaran, Phys. Rev. E 57, 5660 (1998).

[34] V. Kumaran, Phys. Rev. E 59, 4188 (1999).

[35] T. P. C. van Noije, M. H. Ernst, and R. Brito, Physica A 251, 266 (1998).

[36] J. J. Brey, J. W. Dufty, and A. Santos, J. Stat. Phys. 97, 281 (1999).

[37] J. W. Dufty (2001), e-print cond-mat/0108444

[38] P. L. Krapivsky and E. Ben-Naim, J. Phys. A: Math. Gen. 35, L147 (2001).

[39] J. T. Jenkins and S. B. Savage, J. Fluid Mech. 130, 187 (1983).

[40] S. Luding, H. J. Herrmann, and A. Blumen, Phys. Rev. E 50, 3100 (1994).

[41] S. McNamara and J.-L. Barrat, Phys. Rev. E 55, 7767 (1997).

[42] A. Puglisi, V. Loreto, U. M. B. Marconi, and A. Vulpiani, Phys. Rev. E 59, 5582 (1999).

[43] A. Baldassarri, U. M. B. Marconi, A. Puglisi, and A. Vulpiani, Phys. Rev. E 64, 011301 (2001).

[44] S. Luding and O. Strauß, in Granular Gases, edited by T. Pöschel and S. Luding (Springer, Berlin, 2000), Lecture Notes in Physics 564, pp. 389-409.

[45] J. S. van Zon and F. C. MacKintosh (2003), e-print cond-mat/0307644

[46] J. J. Brey and M. J. Ruiz-Montero, Phys. Rev. E 67, 021307 (2003).

[47] A. Barrat and E. Trizac, Granular Matter 4, 57 (2002).

[48] D. Paolotti, C. Cattuto, U. M. B. Marconi, and A. Puglisi, Granular Matter 5, 75 (2003).

[49] S. Luding, Phys. Rev. E 52, 4442 (1995).

[50] S. Luding, E. Clément, A. Blumen, J. Rajchenbach, and J. Duran, Phys. Rev. E 50, R1762 (1994).
[51] R. Ramírez, D. Risso, and P. Cordero, Physical Review Letters 85, 1230 (2000).

[52] S. Luding, E. Clément, J. Rajchenbach, and J. Duran, Europhys. Lett. 36, 247 (1996).

[53] I. Goldhirsch and G. Zanetti, Phys. Rev. Lett. 70, 1619 (1993).

[54] N. V. Brilliantov, F. Spahn, J. M. Hertzsch, and T. Pöschel, Phys. Rev. E 53, 5382 (1996).

[55] O. R. Walton and R. L. Braun, J. Rheol. 30, 949 (1986).

[56] O. Herbst, M. Huthmann, and A. Zippelius, Granular Matter 2, $211(2000)$

[57] V. Kumaran, Phys. Rev. Lett. 82, 3248 (1999).

[58] B. D. Lubachevsky, J. Comp. Phys. 94, 255 (1991).

[59] S. Luding and S. McNamara, Granular Matter 1, 113 (1998).

[60] B. J. Glasser and I. Goldhirsch, Phys. Fluids 13, 407 (2001).

[61] I. Goldhirsch, in Physics of dry granular media, edited by H. J. Herrmann, J.-P. Hovi, and S. Luding (Kluwer Academic Publishers, Dordrecht, 1998), pp. $371-400$.

[62] E. Khain and B. Meerson, Europhys. Lett. 65, 193 (2004).

[63] S. McNamara and S. Luding, Phys. Rev. E 58, 813 (1998).

[64] P. K. Haff, J. Fluid Mech. 134, 401 (1983).

[65] F. J. Rogers and D. A. Young, Phys. Rev. A 30, 999 (1984).

[66] J. P. Hansen and I. R. McDonald, Theory of simple liquids (Academic Press Limited, London, 1986).

[67] S. Luding, Phys. Rev. E 63, 042201 (2001).

[68] R. Soto and M. Mareschal, Phys. Rev. E 63, 041303 (2001).

[69] S. J. Moon, J. B. Swift, and H. L. Swinney, Phys. Rev. E 69, 011301 (2004), e-print cond-mat/0309480

[70] S. J. Moon, M. D. Shattuck, and J. B. Swift, Phys. Rev. E 64, 031303 (2001).

[71] A. Barrat and E. Trizac, Eur. Phys. J. E 11, 99 (2003).

[72] D. Henderson, Molec. Phys. 30, 971 (1975).

[73] The expression (15) for $G$ with $\alpha=1$ is known from the exact equation of state $p /(\rho T)=1+2^{d-1} \phi \chi$ of a $d$-dimensional elastic hard-sphere gas in thermal equilibrium [66]. 
This figure "fig13a.gif" is available in "gif" format from: http://arxiv.org/ps/cond-mat/0402104v2 
This figure "fig13b.gif" is available in "gif" format from: http://arxiv.org/ps/cond-mat/0402104v2 
This figure "fig13c.gif" is available in "gif" format from: http://arxiv.org/ps/cond-mat/0402104v2 
This figure "fig13d.gif" is available in "gif" format from: http://arxiv.org/ps/cond-mat/0402104v2 
This figure "fig13e.gif" is available in "gif" format from: http://arxiv.org/ps/cond-mat/0402104v2 
This figure "fig13f.gif" is available in "gif" format from: http://arxiv.org/ps/cond-mat/0402104v2 
This figure "fig14.gif" is available in "gif" format from: http://arxiv.org/ps/cond-mat/0402104v2 